\title{
Erratum: Phase diagram of the interacting Majorana chain model \\ [Phys. Rev. B 92, 235123 (2015)]
}

Armin Rahmani, Xiaoyu Zhu, Marcel Franz, and Ian Affleck

(Received 12 February 2016; published 4 March 2016)

DOI: 10.1103/PhysRevB.93.119901

The following statement was missing from the acknowledgments: This research was enabled in part by support provided by WestGrid (www.westgrid.ca) and Compute Canada Calcul Canada (www.computecanada.ca). 